\title{
Indemnisation des cas très coûteux avec SwissDRG
}

\author{
Certains cas de traitement stationnaires dans le domaine somatique aigu sont très \\ complexes et très coûteux. Malgré le codage de toutes les affections traitées et de \\ l'ensemble des prestations fournies, il n'est pas possible de définir un DRG qui \\ couvrirait ne serait-ce que de manière approximative les frais engendrés. II en ré- \\ sulte des pertes financières.
}

\section{Petra Ingenpass}

Responsable suppléante du département Tarifs et économie de la santé pour les médecins hospitaliers

La plupart des cas de traitement stationnaires coûteux sont couverts par les DRG. Il en va cependant tout autrement pour les cas très coûteux attribués à un DRG qui prévoit une indemnisation bien trop basse; l'hôpital connaît alors une perte financière.

Un cas cher n'est pas égal à un autre cas cher La définition des cas extrêmement coûteux ou des cas à hauts coûts n'est pas clairement établie. En Alle-

\section{Les hôpitaux universitaires suisses doivent compter avec des pertes de l'ordre de 20000 CHF à 50000 CHF par cas très coûteux.}

Correspondance: Dr Petra Ingenpass FMH

Froburgstrasse 15 4600 Olten Tél. 0313591111 Fax 0313591112

tarife.spital[at]fmh.ch magne, selon la classification du Dr A. Tecklenburg de la Haute école de médecine de Hanovre (MHH), les patients sont définis comme "cas extrêmement coûteux» lorsqu'ils engendrent des frais de traitement supérieurs à 20000 euros, dont moins de la moitié cependant est couverte par le forfait par cas [1]. A la MHH, entre 250 et 450 cas par an répondent à ces critères. Le traitement de ces patients entraîne une perte moyenne de 25000 à 30000 euros par cas, si bien que, selon le Dr A. Tecklenburg, la MHH présente un volume de sous-couverture d'env. 6,5 millions d'euros par an. Si on extrapole ce calcul au territoire fédéral allemand, cela représente un volume global d'environ un demi-milliard d'euros [2].

La Direction de la santé du canton de Zurich fait, elle aussi, état de groupes de patients pour lesquels les frais engendrés dépassent de plus de 30000 francs les revenus obtenus par les forfaits par cas. A l'Hôpital universitaire de Zurich, près de 2000 cas de traitement fortement déficitaires de ce genre ont été traités [3].

SwissDRG SA aborde également le problème des cas à hauts coûts dans son document stratégique [4] sur le développement futur du système SwissDRG. On y donne comme exemple un cas très coûteux de plus de 500000 francs et un écart avec les coûts moyens de plus de 50000 francs. Du point de vue de la FMH, ces deux valeurs sont trop hautes; on ne devrait pas utiliser comme critère un exemple de cas aux coûts globaux de plus d'un demi-million de francs. De tels coûts ne concernent qu'un nombre très limité de cas.

\section{Causes diverses}

Les cas de traitement à hauts coûts avec une souscouverture des frais de traitement ne sont pas rares et on les trouve en particulier, mais pas seulement, dans les hôpitaux universitaires ou les centres spécialisés. Le corps médical a très tôt reconnu la problématique de la sous-couverture des cas très coûteux. Lors de la dernière rencontre organisée par le domaine «Tarifs et économie de la santé pour les médecins hospitaliers» de la FMH, le Prof. Guido Beldi, délégué DRG de la Société suisse de chirurgie viscérale [5], a présenté des exemples concernant sa propre clinique. Ceux-ci montraient clairement que de tels cas à hauts coûts sous-payés ont fréquemment déjà été traités dans un autre hôpital, avec une évolution complexe ayant nécessité un transfert à l'hôpital universitaire. Des réopérations, des soins intensifs et des médicaments ou produits sanguins coûteux étaient souvent indispensables (tabl. 1).

Si l'on considère par exemple l'unité de soins intensifs comme cause possible de coûts élevés, on relève que les traitements de médecine intensive peuvent être pris en compte à l'aide d'un code de procédure

Tableau 1
Causes fréquentes de cas très coûteux:
Transfert d'un autre hôpital
Soins préalables externes
Cas spécialisés et/ou chirurgicaux
Cas complexes avec PCCL élevé [6]
Densité de prestations élevée
Unité de soins intensifs et/ou intermédiaires
Durée de séjour élevée
Médicaments coûteux


ad hoc dans le système SwissDRG 2.0. Un code pour traitement en unité de soins intermédiaires, mobilisant moins de ressources, a été proposé en 2012. Sans ce code de procédures, il n'est pas possible d'avoir une prise en compte appropriée de la prestation et des coûts pour les soins intermédiaires dans la calculation DRG. Cela dit, la sous-couverture des cas de traitement est aussi un problème pour les petits hôpitaux et engendre un déficit considérable, comme Madame Kerstin Schlimbach Neuhauser, internistecadre [7], l'a montré à l'aide de plusieurs cas lors de la même rencontre de la FMH. Hormis les médicaments, les frais médicaux (médecin agréé) et les prestations de tiers y engendrent également des coûts élevés.

Les exemples du Prof. Guido Beldi montrent que, dans un hôpital universitaire, les cas très coûteux occasionnent le plus souvent des pertes de 20000 francs à 50000 francs du fait de leur sous-couverture. Dans

\section{Des cas à hauts coûts sous-payés sont aussi possibles dans un petit hôpital.}

sa clinique, 7,6\% des cas présentent un déficit de plus de 20000 francs. Pour certains cas, le manque à gagner s'élève même à plus de 200000 francs. Dans un petit hôpital de soins de premier recours, les pertes se situent en moyenne entre 15000 et 30000 francs.

\section{Premières approches d'une solution en Allemagne}

En Allemagne, en 2006 déjà, soit deux ans après l'introduction obligatoire des forfaits par cas dans le secteur stationnaire, la sous-couverture de certains cas à hauts coûts a donné lieu à un intense débat et on a cherché des solutions pour résoudre ce problème.

Les hôpitaux qui fournissent les données sur les coûts et les prestations pour l'entretien et le développement des forfaits par cas allemands peuvent saisir séparément les données sur les cas à hauts coûts souspayés. Sur cette base, on a trouvé pour différents DRG et diverses constellations des solutions dans le cadre du système de forfaits par cas. Celles-ci concernent notamment la médecine intensive, les soins aux enfants, les interventions multiples ainsi que les procédures en plusieurs temps. Ainsi, par exemple, on a encore plus différencié les DRG de ventilation artificielle, créé des codes pour les traitements complexes ou introduit, sur la base des données, des répartitions selon l'âge. Il existe par exemple, dans le système DRG allemand, des DRG spécifiques pour des cas chirurgicaux rares et coûteux. La Suisse pourrait profiter de cette expérience en reprenant dans SwissDRG quelques-uns de ces aménagements. Les plus de
150 rétributions additionnelles tarifées et non tarifées [8] disponibles en Allemagne contribuent également à une meilleure prise en compte des prestations fournies. Les rétributions additionnelles contribuent à réduire le volume de sous-indemnisation pour les cas isolés complexes et très coûteux. Simultanément, elles empêchent une distorsion systématique de l'indemnisation des prestations.

En fait, ces solutions représentent des avancées dans la bonne direction, mais elles ne suffisent de loin pas pour réduire les pertes financières engendrées par des groupes de patients déterminés. C'est pourquoi même en Allemagne, où l'on dispose d'une expérience de 10 ans sur les forfaits par cas, on continue de discuter de nouvelles solutions possibles.

\section{La Suisse a besoin de bonnes solutions}

SwissDRG SA observe les nouveaux développements en Allemagne et vérifie s'il serait possible de les reprendre dans le système suisse. En outre, après de nombreuses interventions de la FMH, SwissDRG SA a calculé, sur la base des données, 28 rétributions additionnelles pour la version 3.0 de SwissDRG.

Cette démarche ne suffit toutefois pas; des mesures complémentaires sont nécessaires. Selon SwissDRG SA, des solutions pour la rémunération des cas très coûteux pourraient, le cas échéant, aussi être élaborées en dehors de la structure tarifaire, si une différenciation dans le cadre du système de forfaits par cas s'avérait impossible.

Maintenant, il s'agit pour SwissDRG SA de développer les solutions en question en collaboration avec ses partenaires. La FMH maintiendra son engagement en vue d'obtenir une indemnisation adéquate des cas très coûteux.

\section{Références}

1 Forum d'automne du VUD: Unikliniken auf der Suche nach der gerechten Finanzierung. In: Das Krankenhaus. 2012;11:1177.

2 Présentation «Weiterentwicklung des DRG-Systems», Dr Andreas Tecklenburg, comité des soins aux malades MHH (Medizinische Hochschule Hannover); 2012.

3 Neue Zürcher Zeitung, 17 novembre 2012, «Hochdefizitäre Patienten und teure Kurzlieger».

4 cf. www.swissdrg.org

5 Prof. Guido Beldi, Clinique universitaire de chirurgie viscérale et de médecine, secteur Chirurgie, Hôpital de l'Ile, Berne.

6 PCCL: Patient Clinical Complexity Level; degré de sévérité global de l'état clinique du patient, prenant en considération les complications et les co-morbidités.

7 Kerstin Schlimbach Neuhauser, médecin adjointe du Service de médecine interne, Hôpital d'Einsiedeln.

8 Pour une rétribution additionnelle tarifée, le prix est calculé; en revanche, l'indemnisation des rétributions additionnelles non tarifées est négociée entre les fournisseurs de prestations et les assureurs-maladie. 